\title{
Modified Langmuir-Blodgett deposition of nanoparticles - measurement of 2D to 3D ordered arrays
}

\author{
L. Chitu, P. Siffalovic, E. Majkova, M. Jergel, K. Vegso, S. Luby, I. Capek ${ }^{1}$, A. Satka ${ }^{2}$, J. Perlich ${ }^{3}$, \\ A. Timmann ${ }^{3}$, S. V. Roth ${ }^{3}$, J. Keckes ${ }^{4}$, G. A. Maier ${ }^{5}$
}

Institute of Physics SAS, Dubravska cesta 9, 84511 Bratislava, Slovakia

${ }^{1}$ Polymer Institute Slovak SAS, Dubravska cesta 9, 84236 Bratislava, Slovakia

${ }^{2}$ International Laser Center, Ilkovicova 3, 81219 Bratislava, Slovakia

${ }^{3}$ HASYLAB /DESY, Notkestr. 86, 22603 Hamburg, Germany

${ }^{4}$ Erich Schmid Institute for Materials Science, Jahnstr. 12, A-8700 Leoben, Austria

${ }^{5}$ Materials Center Leoben Forschung GmbH, Roseggerstr. 12, A-8700 Leoben, Austria

\begin{abstract}
The ordered nanoparticle monolayers and multilayers over macroscopic areas were prepared by the modified LangmuirBlodgett method. Using this approach, the nanoparticle monolayer is formed on the water surface by compression and subsequently it is transferred onto the substrate by a controlled removal of the water subphase. The ordering and homogeneity of the prepared mono- and multilayers was studied by scanning electron microscope (SEM), grazing-incidence small-angle $\mathrm{X}$-ray scattering (GISAXS) and X-ray reflectivity (XRR) techniques. From the results it follows that an ordered nanoparticle monolayer was formed over a large area. For the multilayer, the layering and lateral ordering of each layer was confirmed by XRR and SEM performed after the deposition of each nanoparticle layer.
\end{abstract}

Keywords: Langmuir-Blodgett, GISAXS, nanoparticles, self-assembly

\section{INTRODUCTION}

$\mathrm{I}_{\mathrm{N}}^{\mathrm{N}}$ NECENT YEARS, a significant development has been achieved in chemical synthesis of metallic nanoparticles.

At present, a wide range of metallic nanoparticles can be prepared with adequate control over their size and shape. Nanoparticles can serve as building blocks for complex thin film structures. In particular, they self-assemble into ordered arrays - monolayers and multilayers - under specific conditions.

For preparation of such ordered nanoparticle arrays various deposition methods have been elaborated up to now. Among them the Langmuir-Blodgett (LB) method has a potential to control the deposition process over a large area. Up to now, the standard LB method $[1,2]$ has been used to prepare ordered arrays of metallic nanoparticles but with limited success. We applied a modified vertical LB technique which allows an improved control of the homogeneity and ordering of nanoparticle arrays.

In this work, we present the formation of ordered monolayers and multilayers of $\mathrm{Fe}_{2} \mathrm{O}_{3}$ nanoparticle arrays over the large areas using a modified LB technique. The lateral ordering and homogeneity of the $2 \mathrm{D}$ arrays is studied by scanning GISAXS and SEM techniques. The layer structure of multilayer arrays is investigated by XRR and SEM method. The GISAXS technique was used to study lateral correlations of the nanoparticles.

\section{SUBJECT \& Methods}

Iron oxide nanoparticles dispersed into toluene were synthesized by a high-temperature solution phase reaction of metal acetylacetonates $\left(\mathrm{Fe}(\mathrm{acac})_{3}, \mathrm{Co}(\mathrm{acac})_{2}\right)$ with 1,2hexadecanediol, oleic acid and oleylamine in phenyl ether [3]. All chemicals used for the array preparation are of high purity grade purchased from Sigma Aldrich and used without further purification. For the LB deposition toluene was substituted by chloroform in order to increase the evaporation rate of the solvent. The toluene was removed by drying in air at room temperature from $20 \mu \mathrm{l}$ solution. Afterwards, the iron oxide nanoparticles were re-dispersed into chloroform. The Si substrates were pre-cleaned before deposition and subsequently treated by hexamethyldisilazane (HMDS) vapours over the night in order to obtain a hydrophobic terminated surface.

Nanoparticle layers were deposited by a modified LB technique using a computer-controlled Langmuir-Blodgett (LB) trough (Nima Technology). In this method Si substrate was placed horizontally inside the trough before the particles were distributed onto the water subphase. The iron oxide nanoparticles dissolved in chloroform were spread by a microsyringe onto air/water interface of a LB trough. Following equilibration at $18^{\circ} \mathrm{C}$ for $30 \mathrm{~min}$ to allow the chloroform to evaporate, the nanoparticle layer was compressed to a monolayer phase. The surface pressure of nanoparticle arrays was monitored by a Wilhelmy plate. The ordered monolayer was transferred on the Si substrate by a regulated removal of the subphase at a surface pressure of $20 \mathrm{mN} / \mathrm{m}$. The nanoparticle multilayers were prepared using the method described above, adding layer by layer one after another.

The size, shape and local ordering of the arrays were studied by a scanning electron microscope (Leo $1550 \mathrm{HR}$ SEM). The homogeneity of the deposited LB layers over the large areas was verified by the GISAXS technique using a scanning mode at spots separated by $5 \mathrm{~mm}$. The reciprocal space maps were measured at the HASYLAB/DESY synchrotron in Hamburg (Germany) at BW4 beamline [4]. The grazing incident angle of monochromatized X-ray beam $(\lambda=1.38 \AA)$ at the sample surface was $0.7^{\circ}$. The beam size at 
the sample was $65 \times 35 \mu \mathrm{m}^{2}$ in horizontal and vertical direction, respectively. The scattered X-ray radiation was detected by an X-ray CCD camera (marCCD165). The sample-detector distance was $225 \mathrm{~mm}$. The transversal coherence length of the incoming beam is $250 \mathrm{~nm}$. This transversal coherence length is projected in one dimension to $20 \mu \mathrm{m}$ due to $0.7^{\circ}$ of grazing incidence angle. The final intensity of the GISAXS pattern is an incoherent sum of scattered radiation from $0.25 \times 20 \mu \mathrm{m}^{2}$ illuminated domains. The GISAXS is sensitive to inhomogeneity in the substrate coverage by the nanoparticles within $0.25 \times 20 \mu \mathrm{m}^{2}$ domains. On the other hand, large-scale inhomogeneities comparable with the X-ray beam finger print on the sample may be detected in the scanning mode regardless the coherence length. The XRR measurements were done on an X-ray diffractometer (Bruker D8 Discover) equipped with a $\mathrm{Cu}$ $\mathrm{X}$-ray rotating anode.

\section{RESUlTS \& DisCUSSION}

The SEM micrograph of sample surface shown in Fig.1a shows a homogenous 2D ordered array of iron oxide nanoparticles prepared by a modified LB deposition. From the detailed SEM image in Fig.1b we observe a typical ordered domain structure of the nanoparticle monolayer [5]. The average particle size of $6.4 \pm 0.6 \mathrm{~nm}$ and the average interparticle distance center-to-center of $7.4 \pm 0.6 \mathrm{~nm}$ were determined from the SEM micrographs and are in good agreement with the transmission electron microscopy (TEM) and X-ray diffraction (XRD) results [6].
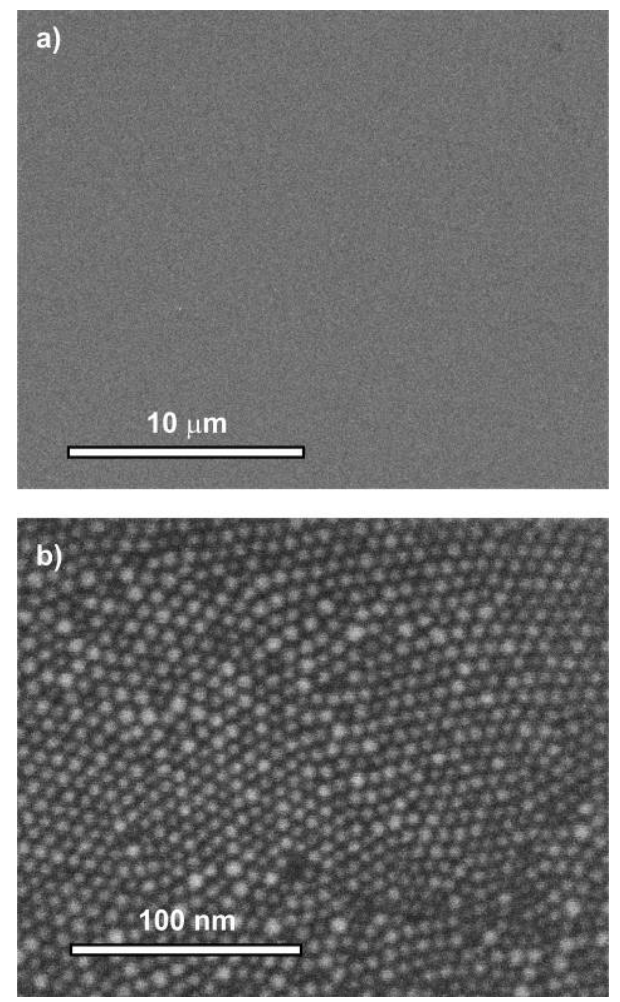

Fig.1. SEM micrographs of the nanoparticle monolayer taken at (a) low and (b) high magnification.
The ordering of the arrays over the large surface area was studied by the GISAXS technique. This method $[7,8]$ provides the statistical averages of the parameters describing the lateral and vertical ordering of the nanoparticles in arrays over macroscopic areas. The GISAXS reciprocal space map of a 2D ordered array of iron oxide nanoparticles deposited on a silicon substrate is shown in Fig. $2 \mathrm{a}$. The $q_{\mathrm{y}}$ and $q_{\mathrm{z}}$ components of the scattering vector are parallel and perpendicular to the substrate surface, respectively. The side maxima on the reciprocal space map indicate the lateral ordering of the nanoparticle monolayer [6]. The uniformity of the deposited nanoparticle monolayer was studied by GISAXS technique in a scanning mode. The horizontal intensity cuts at the critical exit angles $\left(q_{\mathrm{z}}=0.69 \pm 0.02 \mathrm{~nm}^{-1}\right)$ of GISAXS patterns for the six different representative locations on the sample surface are shown in Fig. 2b. The variations in the scattered X-ray intensity between the different locations at the sample surface are less than 5\% and provide evidence for a large scale homogeneous deposition of nanoparticle arrays using a modified LB technique.
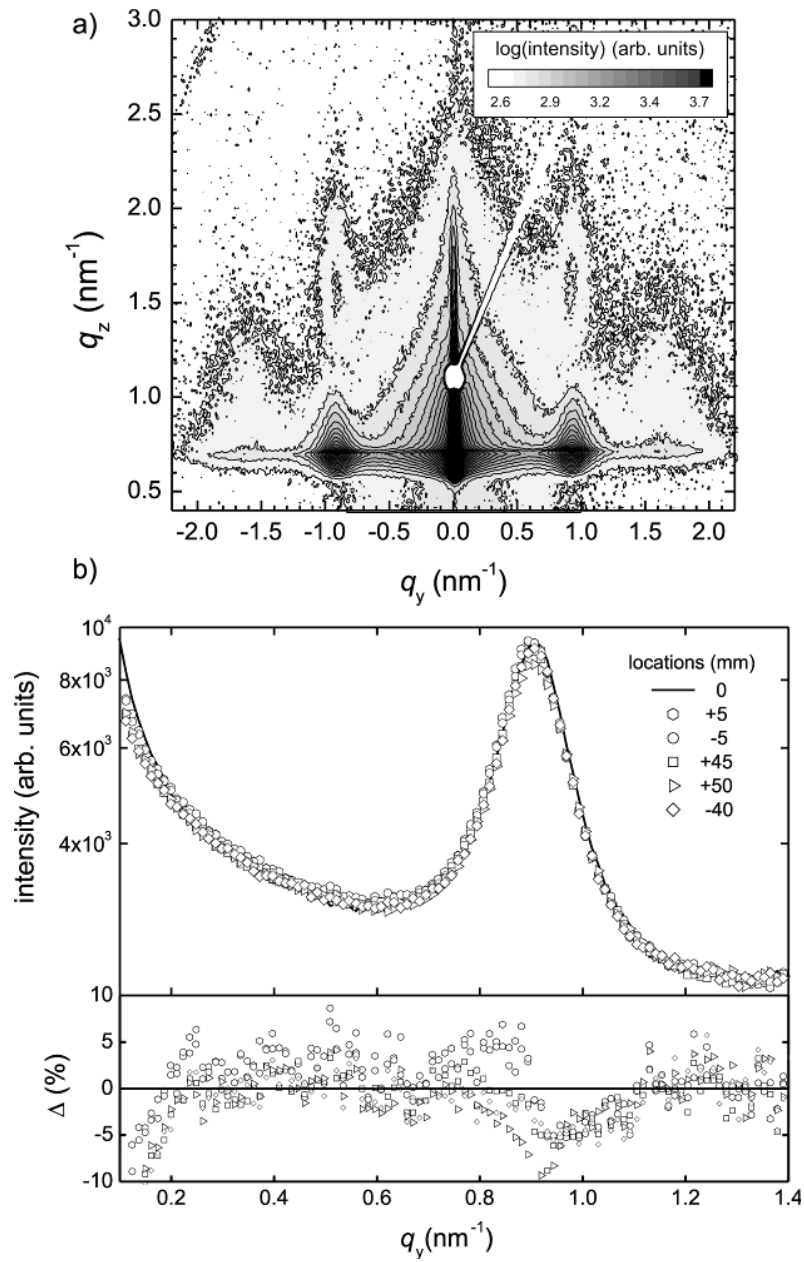

Fig.2(a). The GISAXS pattern of a nanoparticle monolayer. (b) Intensity profile of scattered X-ray radiation at the critical exit angle for six different positions on the sample surface. 
Employing the same modified LB method the multilayer arrays of nanoparticles were prepared. The layering of the arrays was studied by XRR measurements. The XRR measurements were performed after the deposition of each layer of nanoparticles. Here we present the XRR measurements of a monolayer and multilayer composed of six layers of nanoparticles. The XRR measurement and simulation of a nanoparticle monolayer are shown in Fig. 3, while the electron density profile extracted by modelling the experimental data is shown in the inset of the graph. The XRR data of the iron oxide nanoparticle monolayer show a series of Kiessig fringes in the reflectivity curve [9]. They arise from the interferences of the X-ray beams reflected at the interfaces between the air, nanoparticle monolayer and silicon substrate. In order to fit the Kiessig fringes observed in the XRR curve we used a model system based on the following layers: $\mathrm{Si}, \mathrm{SiO}_{2}, \mathrm{HMDS}$ and nanoparticle layer. The nanoparticle layer was modelled as a three component layer composed of two organic layers (OL1 and OL2) representing the surfactant molecules capping the inorganic nanoparticle core denoted as the third layer $(\mathrm{FeO})$ in the simulation. The surfactant molecules prevent the agglomeration of the nanoparticles. The reflectivity curve was fitted by a genetic algorithm modified for XRR applications [10]. The layer thickness, roughness and density obtained from the XRR simulations are presented in Tab.1.

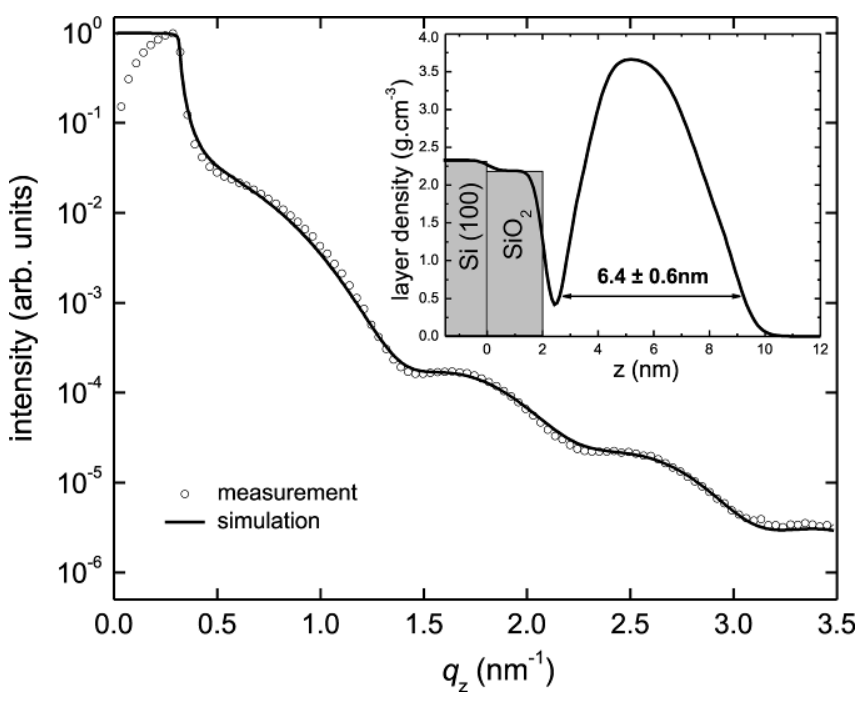

Fig.3. The measured and simulated X-ray reflectivity for a nanoparticle monolayer. The inset shows simulation based density profile.

The reconstructed layer density profile (inset in Fig.3) based on the values from Tab. 1 exhibits a continuous monolayer with a thickness of the nanoparticle diameter, confirming the presence of a closed nanoparticle monolayer stemming from the SEM and GISAXS analyses.

The deposited nanoparticle multilayer composed of six layers was studied by XRR. The XRR measured curve (Fig.4) of the 3D nanoparticle array shows a series of Kiessig fringes and Bragg peaks which indicate well defined vertical layering of the nanoparticle sheets. In the XRR simulation the previous monolayer model was extended by additional five layers with the composition identical to a single monolayer (OL1/FeO/OL2). During the fitting procedure the thickness and density of the five additional layers were kept constant. The results obtained from the density profile indicate that six uniform layers of nanoparticles with the particle diameter of approximately $6.4 \mathrm{~nm}$ were deposited over the large area of the $\mathrm{Si}$ substrate.

Table 1 Summary of parameters obtained by fitting the XRR curve.

\begin{tabular}{|c|c|c|c|c|}
\hline & HMDS & OL1 & FeO & OL2 \\
\hline thickness $(\mathrm{nm})$ & 1.3 & 1.2 & 4.2 & 1.5 \\
\hline roughness $(\mathrm{nm})$ & 0.84 & 0.61 & 0.95 & 0.96 \\
\hline density $(\mathrm{g} / \mathrm{cm} 3)$ & 1.2 & 2 & 3.7 & 2 \\
\hline
\end{tabular}

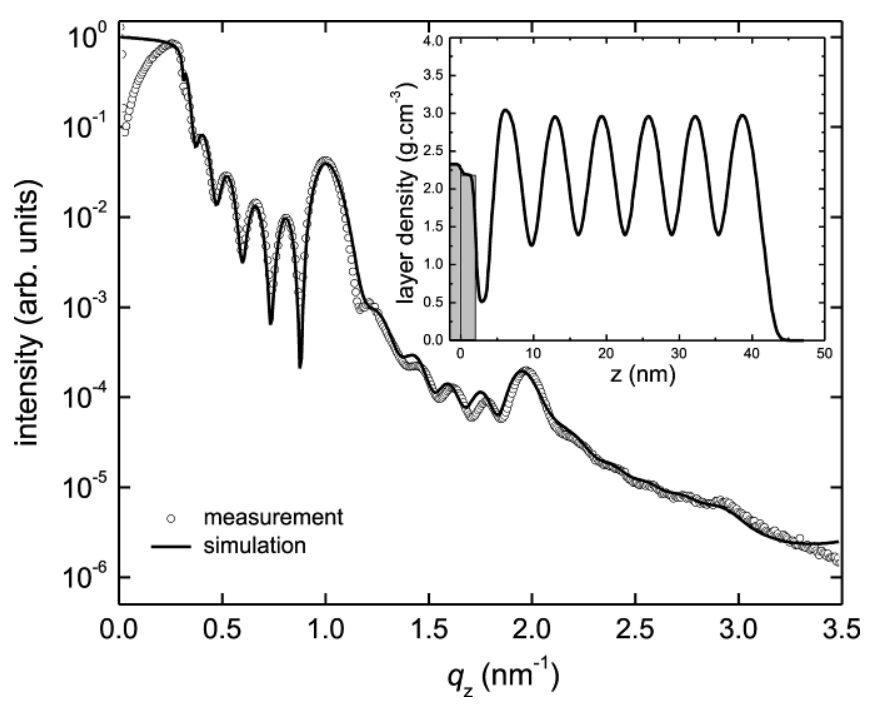

Fig.4. The measured and simulated X-ray reflectivity for six layers of nanoparticles. The inset shows simulation based density profile.

\section{CONCLUSION}

The present results demonstrate the possibility of deposition of ordered nanoparticle mono- and multilayers using a modified LB technique onto large area substrates. The local ordering of the nanoparticle arrays was studied by the SEM micrographs and proved the formation of the typical ordered domain structure. The scanning GISAXS technique showed the formation of ordered and uniform nanoparticle 2D arrays over a large area $\left(20 \mathrm{~cm}^{2}\right)$ which was also confirmed by XRR. The distinct Bragg reflection peaks observed in the XRR curves for a nanoparticle multilayer confirmed the formation of a periodic layered structure. From XRR and GISAXS one can conclude lateral ordering of nanoparticles within each deposited layer.

\section{ACKNOWLEDGMENT}

This publication is the result of the project implementation: Centre of Excellence for New Technologies in Electrical Engineering, ITMS code 26240120011, supported by the Research \& Development Operational Programme funded by the ERDF. 


\section{REFERENCES}

[1] Lee, D.K., Kim, Y.H., Kim, C.W., Cha, H.G., Kang, Y.S. (2007). Vast magnetic monolayer film with surfactant-stabilized $\mathrm{Fe} 3 \mathrm{O} 4$ nanoparticles using Langmuir-Blodgett technique. J. Phys. Chem. B, 111, 9288-93.

[2] Brown, J.J., Porter, J.A., Daghlian, C.P., Gibson, U.J. (2001). Ordered arrays of amphiphilic gold nanoparticles in Langmuir monolayers. Langmuir, 17, 7966-9.

[3] Chitu, L., Jergel, M., Majkova, E., Luby, S., Capek, I., Satka, A., Ivan, J., Kovac, J., Timko, A. (2007). Structure and magnetic properties of $\mathrm{CoFe} 2 \mathrm{O} 4$ and Fe3O4 nanoparticles. Mat. Sci. Eng. C: Bio. S., 27, 1415-7.

[4] Roth, S.V., Döhrmann, R., Dommach, M., Kuhlmann, M., Kröger, I., Gehrke, R., Walter, H., Schroer, C., Lengeler, B., Müller-Buschbaum, P. (2006). Smallangle options of the upgraded ultrasmall-angle $\mathrm{X}$-ray scattering beamline BW4 at HASYLAB. Rev. Sci. Instrum., 77, 085106.
[5] Chushkin, Y., Chitu, L., Halahovets, Y., Luby, S., Majkova, E., Satka, A., Leo, G., Giersig, M., Hilgendorff, M., Holy, V., Konovalov, O. (2006). GISAXS studies of self-assembling of colloidal Co nanoparticles. Mat. Sci. Eng. C: Bio. S., 26, 1136-40.

[6] Siffalovic, P., Majkova, E., Chitu, L., Jergel, M., Luby, S., Satka, A., Roth, S.V. (2007). Self-assembly of iron oxide nanoparticles studied by time-resolved grazing-incidence small-angle x-ray scattering. Phys. Rev. B, 76, 1954321-8.

[7] Levine, J.R., Cohen, L.B., Chung, Y.W., Georgopoulos, P. (1989). Grazing-incidence smallangle X-ray scattering - new tool for studying thinfilm growth. J. Appl. Crystallogr., 22, 528-32.

[8] Renaud, G., Lazzari, R., Leroy, F. (2009). Probing surface and interface morphology with Grazing Incidence Small Angle X-Ray Scattering. Surf. Sci. Rep., 64, 255-380.

[9] Kiessig, H. (1931). Interferenz von Röntgenstrahlen an dünnen Schichten. Ann. Phys. (Leipzig), 10, 769-72.

[10] Ulyanenkov, A., Sobolewski, S. (2005). Extended genetic algorithm: application to X-ray analysis. $J$. Phys. D: Appl. Phys., 38, A235-A8.

Received September 28, 2010. Accepted December 17, 2010. 